\title{
Differences in Falls and Recovery from a Slip Based on an Individual's Lower Extremity Corrective Response
}

Samuel J. Wilson ${ }^{1 *}$, Paul T. Donahue ${ }^{2}$, Charles C. Williams ${ }^{3}$, Christopher M. Hill ${ }^{4}$, Jeffrey D. Simpson ${ }^{5}$, Dwight E. Waddell ${ }^{6}$, Jeremy P. Loenneke ${ }^{7}$, Harish Chander ${ }^{8}$, Chip Wade ${ }^{9}$, John C. Garner ${ }^{10}$

${ }^{1}$ Department of Health Sciences and Kinesiology, Georgia Southern University, Statesboro, GA, 30460,

${ }^{2}$ School of Kinesiology and Nutrition, University of Southern Mississippi, Hattiesburg, MS

${ }^{3}$ Department of Exercise Science, LaGrange College, Lagrange, GA

${ }^{4}$ Department of Physical Education, Northern Illinois University, DeKalb, IL

${ }^{5}$ Department of Exercise Science and Community Health, University of West Florida, Pensacola, FL

${ }^{6}$ Department of Biomedical Engineering, The University of Mississippi, University, MS

${ }^{7}$ Department of Health, Exercise Science, and Recreation Management, The University of Mississippi, University, MS

${ }^{8}$ Department of Kinesiology, Mississippi State University, Mississippi State, MS

${ }^{9}$ Department of Industrial \& Systems Engineering, Auburn University, Auburn, AL

${ }^{10}$ Department of Kinesiology and Health Promotion, Troy University, Troy, AL

Corresponding Author: Samuel J. Wilson, E-mail: sjwilson@georgiasouthern.edu

\section{ARTICLE INFO}

Article history

Received: 29-04- 2019

Accepted: 28-06- 2019

Published: 31-07- 2019

Volume: 7 Issue: 3

Conflicts of interest: None

Funding: None

\begin{abstract}
Background: Slips and falls account for high rates of injury and mortality in multiple populations. The corrective responses during the slip perturbation have been well documented. However, when a fall results from a slip, it is unclear which of these responses were inadequate. Objective: The purpose of this study was to examine differences in lower extremity corrective responses of the slip recovery response between individuals who fall and those who recover. Methodology: Sixtyfour participants completed this study ( 32 males \& 32 females). Participant's gait kinematics and kinetics were collected during normal gait (NG) and an unexpected slip (US). A prediction equation for slip outcome and slip severity were created using a binary logistic regression model. Results: Our findings show an increased time to peak hip extension (OR $=1.006, \mathrm{CI}$ : 1.000 $1.011)$ and ankle dorsiflexion $(\mathrm{OR}=1.005, \mathrm{CI}$ : 1.001-1.009) moments increased the odds of falling, while the average ankle moment was negatively associated with falling $(\mathrm{OR}=0.001$, CI: 0.001-0.005). Conclusions: Rapid lower extremity corrective responses appear critical in arresting the slip and preventing a fall. While there are various strategies for slip recovery, our findings suggest that the primary recovery mechanism at the slipping hip may play a vital role in preventing the fall.
\end{abstract}

Key words: Accidental Falls, Gait, Logistic Models, Lower Extremity

\section{INTRODUCTION}

Slips and falls are a major cause of injury and death in the United States (Burns, Stevens, \& Lee, 2016; Chambers \& Cham, 2007). Injuries due to falls are the leading cause of non-fatal injuries and the third leading cause of fatal injuries in the United States, but more importantly, these injury incidence rates have been increasing in recent years (Sise et al., 2014). Specifically, in $2010,33 \%$ of all medically consulted injuries were due to falls, whereas is $2017,37 \%$ were due to falls Adams et al., 2011). The Global Burden of Disease Study 2010 reported that between 1990 and 2010 falls increased in rank from the $24^{\text {th }}$ to the $15^{\text {th }}$ leading cause of United States disability-adjusted life years (DALYs), with over a $50 \%$ increase in DALYs. To put this in perspective, the CDC reported every 11 seconds, an older adult is treated in the emergency room for a fall; every 19 minutes, an older adult dies from a fall. From an economic perspective, in 2015, the total healthcare spending attributable to falls was more than $\$ 50$ billion (Florence et al., 2018). From an occupational setting, despite efforts being made to mitigate fall-related injuries through Occupational Safety and Health Administration (OSHA) guidelines (Chang, Leclercq, Lockhart, \& Haslam, 2016), or protective efforts to decrease slip propensity, such as footwear (Chander, Garner, \& Wade, 2015b, 2016), costs of fall-related injuries are still increasing. The total cost stated above for fall-related injuries in 2012 was approximately $\$ 16.48$ billion, which increased in 2013 to approximately $\$ 17.92$ billion, and increased further in 2014 to about $\$ 18.42$ billion (Liberty Mutual Research Institute for Safety, 2014; Liberty Mutual Research Institute for Safety, 2016; Liberty Mutual Research Institute for Safety, 2017). Considering this increasing trend in fall-related injuries,

Published by Australian International Academic Centre PTY.LTD.

Copyright (c) the author(s). This is an open access article under CC BY license (https://creativecommons.org/licenses/by/4.0/) http://dx.doi.org/10.7575/aiac.ijkss.v.7n.3p.34 
along with the evidence to suggest slipping is a main cause of falls despite increased safety mandates, the need for further understanding the slip recovery process itself is immense.

Researchers have commonly classified slips across a range of slip severities based on the magnitude of heel slip distance, as well as heel slip velocity (Cham \& Redfern, 2001; Chander et al., 2015b; Redfern et al., 2001). A common set of cutoff values was provided by Strandberg and colleagues (1981) and classified slips into mini slips, where participants did not detect the slipping motion, midi-slips, where the slip resulted in a recovery without major gait disturbances, and maxi-slips, in which there was a recovery but with large corrective responses (Strandberg \& Lanshammar, 1981). Recent work has attempted to quantify the slip response as a fall or recovery through the use of a force criterion in the fall arrest harness system (Sawers, Pai, Bhatt, \& Ting, 2017; Yang \& Pai, 2011).

Previous research using latencies alone from the Motor Control Test (MCT) on the NeuroCom ${ }^{\circledR}$ suggests that individuals with slower reaction times slip longer than those with faster latencies (Lockhart, 2008). While literature examining corrective kinetic responses has suggested a stereotyped reflexive response that involves flexion of the knee, and extension of the hip in the leading leg (Cham, 2001), these responses have been reported to be scaled to the severity of the slip, but have yet to be analyzed between groups of fallers and those who recover after an induced slip, or specifically between groups of slip types. We suggest using a specific classification of slip severity, as well as an objective assessment of fall/recover. These measures may provide more insight into the role of reaction time responses to a slip, as well as the contribution of individual recovery responses between people who experience more severe slips.

To reduce fall risks and improve balance and mobility, several types of training modalities have been previously used (Kosma, Hondzinski, \& Buchanan, 2017; Parijat \& Lockhart, 2012; Sadeghi et al., 2017)Perturbation training is an emerging paradigm to reduce falls (Lee, Bhatt, Liu, Wang, \& Pai, 2018), with recent work demonstrating reduced fall outcomes when exposed to a slip as well as improved stability measures. Further, in addition to improved slip outcomes, slip training has shown improvements in reactive slip reactions in both young, and old adults (Parijat \& Lockhart, 2012). With perturbation training showing promising results with regards to slip outcomes, and slip recovery responses across young and older adults, examining which recovery measures distinguish between falling and recovering during an over-ground slip may aid in the application of this training. Thus, the purpose of this study was to examine slip recovery responses of the lead slipping leg and their associated odds of falling after an induced slip perturbation. To examine this, slips were classified two separate ways, according to slip severity, and whether or not the slip resulted in a fall. We hypothesize that those who experience a greater level of hazardous slip will have decreased reaction time latencies, and will exhibit slower corrective responses of the slipping leg. Similarly, we hypothesize that those classified as "fallers" during the slip trial will have decreased reaction times and response measures compared to those who were classified as "recoveries".

\section{METHODOLOGY}

\section{Participants and Study Design}

This study followed a cross sectional, between subjects design to analyze relationships between slip recovery kinetics, and slip outcomes. Two separate logistic regression models were created, with dependent variables in the first and second models being slip outcome (fall vs recovery) and slip severity (hazardous vs non-hazardous), respectively. Independent variables of interest included reaction time latencies to a postural perturbation, and recovery kinetics of the slipping leg, specifically, average joint moments, peak joint moments, and time to peak joint moments of the ankle, knee, and hip. One hundred healthy participants were recruited (50 male, 50 female; age: $21 \pm 3$ years; height: $171.3 \pm 13.7 \mathrm{~cm}$; weight: $75.6 \pm 16.5 \mathrm{~kg}$ ). Participants were excluded if they had any history of musculoskeletal injuries, cardiovascular, neurological, or vestibular disorders, or any inability to walk and stand without support. Participants were also excluded from analysis if they missed contact with the force plate during the unexpected slip trial, had marker dropout which impeded calculation of slip parameters, or experienced toe-off slips at the end of stance phase instead of heel-strike. This yielded a final sample, of 64 participants ( 32 male, 32 female; age: $21.82 \pm 3.14$ years; height: $171.07 \pm 14.91 \mathrm{~cm}$; weight: 75.78 $\pm 16.48 \mathrm{~kg}$. All participants were informed of the experimental procedures as well as any potential risks of the study before giving written informed consent approved by the University's Institutional Review Board. Participants were also asked to complete a physical activity readiness questionnaire (PAR-Q) to address the aforementioned health-related exclusionary criteria. Sample size estimation was based on previous research done in the Applied Biomechanics Laboratory at the University of Mississippi and an approximated 30\% fall rate among participants, to allow for the model building of approximately 3 predictor variables, using an alpha level of 0.05 .

\section{Experimental Procedures}

All participants visited the testing laboratory for two visits, one being a familiarization day, and the next being an experimental day. The first visit served as an administrative day where participants were screened for exclusionary criteria through preliminary paperwork. Following paperwork, participants had a variety of anthropometrics measured, including; height, weight, leg lengths, knee and ankle widths, and shoe size. Once anthropometrics were obtained, participants were familiarized with the experimental protocol, including completing a full session of the motor control test (MCT). Finally, participants were strapped into the harness and allowed to experience the normal walking protocol across the laboratory while wearing the harness.

\section{Experimental Testing}

Testing began with participants completing the MCT on the NeuroCom. Next, participants were then strapped in the harness system and had a series of practice gait trials across the 
vinyl floored testing surface under dry nonslip conditions to get accustomed with the gait trials and the testing environment at a self-selected speed of walking. These practice gait trials were also used to make sure that the participants strike the center of the force plate with both their feet, unintentionally at their normal walking pattern and pace and to avoid any intentional modification of their step lengths during the data collection procedure. Following the initial practice gait trials, data collection was done for five normal dry gait trials with no breaks or stops between the gait trials with the instruction "walk as normally as possible with the same speed". With the completion of the fifth normal dry gait trial, participants still walked with the same pattern and speed, but at the end of all further gait trials, the participants took 30-45 second breaks facing away from the walking surface and listened to music played on noise-cancellation headphones, which would take away knowledge of the potential slip trial.

Following a repeated number of gait trials under normal dry conditions, one particular trial was chosen randomly to be the unexpected slip (US) trial and the contaminant was applied to the force plate without the participant's knowledge. Participants were still given the same walking instruction to ensure that the walking trial will be treated as an unexpected slip event. On completion of the US, the force plate was cleaned with a dry-wet vacuum and soap and water and dried completely. These methods of providing an unexpected slip perturbation have been used by our group (Chander, Garner, \& Wade, 2015a), and others (Cham \& Redfern, 2001; Chambers \& Cham, 2007), with minimal gait modifications between normal dry gait trials and unexpected slip trials.

\section{Instrumentation}

Vicon Nexus (Oxford, UK) 3D motion capture system with 8 infra-red T-series cameras was used to collect and analyze kinematic gait data. A lower body plug-in gait model from the Helen-Hayes marker system was used for the participant configuration and the kinematic data was sampled at $100 \mathrm{~Hz}$ and collected using the Vicon Nexus software.

Two force plates, Bertec (Bertec Corporation, Columbus, $\mathrm{OH}$ ) and AMTI (AMTI Force and Motion, Watertown, MA) embedded into the floor were used to quantify ground reaction forces. Force plate data is collected through the Vicon Nexus system as an analog device and sampled at 1,000 Hz.

Reaction time latencies were assessed using the Motor Control Test (MCT) on the NeuroCom Equitest System ${ }^{\mathrm{TM}}$ (NeuroCom International, Inc. Clackamas, Oregon). The system uses an 18 " x 18 " dynamic dual force plate, that can translate in the backward and forward directions to create three testing conditions, which include backward translations [small (BWS)/medium (BWM)/large (BWL)] and forward translations [small (FWS)/medium (FWM)/large (FWL)] (Nashner 1993). Response latencies in milliseconds (ms) are then provided for the backward small (BWS), medium (BWM), and large (BWL), and forward small (FWS), forward medium (FWM), and forward large (FWL) conditions. A detailed description of these balance tests is explained elsewhere (Nashner 1993, Guskiewicz and Perrin 1996).
A uni-track fall arrest system from Rigid Lines (Millington, TN) capable of supporting up to $900 \mathrm{lb}$ was used to prevent any falls onto the flooring surface. Participants were strapped into a harness system connected to a moveable trolley inside the fall arrest track. The fall arrest track and the harness along with the trolley are connected by a pulley system that allows the investigators to move the trolley above the participant during walking trials so the participant does not lead the trolley and the trolley does not lead the participant. Finally, an electronic crane scale was attached between the harness, and trolley system in order to quantify bodyweight bared by the harness system during walking trials.

A 3:1 mixture of vegetable-based glycerol and water was used as the slippery contaminant. This ratio of glycerol and water has been used previously by our lab (Chander et al., 2015a, 2015b), as well as others (Cham \& Redfern, 2001; Chambers \& Cham, 2007). During slip trials, glycerol was applied and evenly distributed on the Bertec force plate, which is the force plate all participants struck with their left leg, regardless of limb dominance. Application of the contaminant was always be performed by the primary investigator using the same measured container in an attempt to minimize errors due to inter- and intra-rater reliability.

\section{Data Analysis}

Kinetics, kinematics, and lower extremity moments were analyzed using the Vicon Nexus software. Raw kinematic data were cleaned removing unlabeled markers, marker gaps were filled using a spline fill and edited to have two complete gait cycles starting with the right leg. The analog kinetic force plate data were filtered using a Butterworth fourth-order filter with zero lag with a cut off frequency of $300 \mathrm{~Hz}$, while the kinematic data were filtered using a Butterworth fourth-order filter with zero lag at $15 \mathrm{~Hz}$ and exported as Excel files for further analyses. Vicon Nexus software was used to determine the moment of heel strike and toe-off of the left leg during the gait trials to determine the stance phase beginning and end. Stance phase was scaled to $100 \%$ (mean stance duration was $748.31 \pm 76.37 \mathrm{~ms}$ ) and average joint moments were calculated throughout stance. Specifically, ensemble averages were calculated for the ankle (average ankle moment [Ank_Avg]), knee (average knee moment [Knee_Avg]), and hip (average hip moment [Hip_Avg]). Additionally, reactive joint moments were quantified using peak moment and time to peak moment. Peak moment was defined as the maximum moment magnitude from heel-contact (HC) to toe-off of the slipping foot for the ankle (peak dorsiflexion moment [Ank_DFp]/peak plantarflexion moment [Ank_PFp]), knee (peak flexion moment [Knee_Fp]/ peak extension moment [Ep]), and hip (peak flexion moment [Hip_Fp]/peak extension moment [Ep]). Time to peak moments was defined as the ratio between the peak moment and the time from $\mathrm{HC}$ to the instance of peak moment, representing the speed of peak moment generation. These time to peak moments were calculated for the ankle (Dorsiflexion time to peak moment [Ank_DFttp]/Plantarflexion time to peak moment [PFttp]), knee (Flexion time to peak moment [Knee_Fttp]/Extension time to peak moment [Ettp]), and hip 
(Flexion time to peak moment [Hip_Fttp]/Extension time to peak moment [Ettp]).

Each slip trial was classified based on two criteria; slip severity, and slip outcome. The outcome of a slip was classified as a fall based on the weight measured by the crane scale during the slip, and was classified as a fall if the peak weight in the scale during the slip trial exceeded $30 \%$ of the participant's body weight (Sawers et al., 2017; Yang \& Pai, 2011). Falls were confirmed by visual inspection. The slip outcome was classified as a recovery if the slip distance was greater than $10 \mathrm{~mm}$ and mean heel slip velocity was greater than $100 \mathrm{~mm} / \mathrm{s}$, but the weight on the harness was less than $30 \%$ of body weight during the slip. Slip trials were also classified based on slip severity as determined by heel slip distance (HSD) (mm) and the mean heel slip velocity (MHSV) $(\mathrm{mm} / \mathrm{s})$ during the first $120 \mathrm{~ms}$ following heel strike of the left leg. The left heel marker was used to determine HSD and MHSV, while the ground reaction forces from the force plate were used to determine the heel strike, using Vicon Nexus software. HSD is defined as the horizontal distance traveled by the left heel marker from the moment of heel-strike to $120 \mathrm{~ms}$ into the gait cycle. MHSV is the average of the horizontal velocity of the left heel marker after the foot strikes the floor and until 120 $\mathrm{ms}$ into the gait cycle. During unexpected slips, HSD and MHSV were classified as non-hazardous (10-30 mm HSD \& MHSV $100-300 \mathrm{~mm} / \mathrm{s}$ ), and hazardous slips (HSD: $>30 \mathrm{~mm} \&$ MHSV: $>300 \mathrm{~mm} / \mathrm{s}$ ) (Chander, Wade, Garner, \& Knight, 2016; Strandberg \& Lanshammar, 1981). Once classified, MCT latencies, and lower body kinetics were analyzed across the established groups.

\section{Statistical Analysis}

Two binary logistic regression models were created using the recovery parameters, and MCT latencies as covariates, and fall or recovery, or slip severity as the dependent outcome variables. Each predictor variable was entered individually into a logistic regression model to obtain unadjusted estimates (Table 1). Next, using Wald scores from the unadjusted model, variables were included in the final model using a purposeful selection method suggested by Hosmer and Lemeshow (Bursac, Gauss, Williams, \& Hosmer, 2008) if their Wald statistics were significant at the $p=0.25$ level initially. Then variables were eliminated one at a time if they were not significant in the multivariate model at the $p=0.1$ level, and when taken out, did not change any remaining parameter estimates by more than $20 \%$. Next, those parameters from the first model were iterated through the final model, to determine which set of covariates provided the best classification. The three variables that classified the highest percentage correctly were included in the final model. Lastly, this final model was tested for multi-collinearity and model fit using individual variance inflation factors (VIF) $<10$, and the Hosmer and Lemeshow goodness of fit test, respectively, and entered concurrently if no collinearity or poor fit was found (Table 2). Nagelkerke's $\mathrm{R}^{2}$ was calculated as an estimate of effect size for the regression model. Statistical significance was established at $p<0.05$.
In addition, independent samples t-tests were performed for each recovery variable between the primary sets of groups (slip outcome [fall vs recover] \& slip severity [non-hazardous vs hazardous]). Statistical significance for these independent samples t-tests were set at $p<0.05$.

\section{RESULTS}

After exclusionairy criteria, analyses were performed on a final sample of 64 participants (32 male, 32 female; age: 21.82 \pm 3.14 years; height: $171.07 \pm 14.91 \mathrm{~cm}$; weight: $75.78 \pm$ $16.48 \mathrm{~kg}$.

\section{Model 1: Fall Status}

After exclusions, the final analysis included 64 participants. For model 1, this included 39 trials classified as recoveries and 25 trials classified as fallsThe results of the independent t-tests between falls and recoveries suggest several differences in lower extremity corrective responses between groups. For the average ankle moment during stance phase, we observed a significant increase in the recovery group compared to the fallers $(\mathrm{t}(63)=2.695, p=0.009)$. Also at the ankle, we saw a significant increase in the time to peak ankle dorsiflexion moment for falls compared to recoveries $(\mathrm{t}(63)=-2.709, p=0.009)$. Finally, at the hip, our data show an increase in the time to peak extension moment about the $\operatorname{hip}(\mathrm{t}(63)=-2.274, p=0.026)$.

Unadjusted logistic regression coefficients are shown in Table 1. These estimates, along with the findings from the t-tests above were used in our model-building approach. In Table 2, the multivariable logistic regression association between the recovery parameters and odds of falling are shown. This final multivariable logistic regression classified recoveries, and falls correctly $92.3 \%$, and $72.0 \%$, respectively $\left(\mathrm{X}^{2}=31.72, p<0.001\right.$, Nagelkerke $\left.\mathrm{R}^{2}=0.530\right)$. This model suggests that an increase in the average ankle moment over the stance phase of the slip trial is associated with decreased odds of falling. While increases in the hip extension time to peak moment and ankle dorsiflexion time to peak moment were associated with increased odds of falling (Table 2).

\section{Model 2: Slip Severity}

Model 2 included 64 participants in the final analysis. These consisted of 37 non-hazardous slips and 27 hazardous slips. For the group comparisons, independent t-tests revealed differences in recovery responses between those who experience non-hazardous slips, and hazardous slips. Similarly to model 1, we observed a significant increase in the average ankle moment in non-hazardous slips compared to hazardous $(\mathrm{t}(62)=3.197, p=0.002)$. Also at the ankle, a significantly increased plantarflexion peak moment was observed in non-hazardous slips compared to hazardous $(\mathrm{t}(62)=2.257$, $p=0.028)$. At the hip, increased time to peak hip extension moment was observed in the hazardous slip group compared to non-hazardous $(\mathrm{t}(62)=-2.120, p=0.038)$. Other variables of interest, while not statistically significant, were the time to peak knee extension $(p=0.09)$ which was decreased in 
Table 1. Binary logistic regression unadjusted estimates examining the association between recovery parameters and the odds of falling after an induced slip

\begin{tabular}{|c|c|c|}
\hline & \multicolumn{2}{|c|}{$\begin{array}{c}\text { Odds ratio }(95 \% \mathrm{CI}) \text { for } \\
\text { falling after the induced slip } \\
\text { perturbation }{ }^{\dagger}\end{array}$} \\
\hline & Odds ratio $(95 \% \mathrm{CI})$ & $\mathbf{p}$ \\
\hline \multicolumn{3}{|l|}{ Recovery Response } \\
\hline Average Ankle Moment & $0.01(0.01-0.49)$ & 0.001 \\
\hline Average Knee Moment & $1.08(0.34-3.38)$ & 0.89 \\
\hline Average Hip Moment & $0.88(0.47-1.65)$ & 0.70 \\
\hline Ankle DF Peak & $0.88(0.45-1.71)$ & 0.71 \\
\hline Ankle PF Peak & $0.06(0.01-0.54)$ & 0.01 \\
\hline Knee Flexion Peak & $2.05(0.62-6.69)$ & 0.23 \\
\hline Knee Extension Peak & $1.04(0.67-1.60)$ & 0.85 \\
\hline Hip Flexion Peak & $1.19(0.88-1.63)$ & 0.25 \\
\hline Hip Extension Peak & $0.80(0.56-1.14)$ & 0.22 \\
\hline Ankle Dorsiflexion TTP & $1.005(1.000-1.009)$ & 0.03 \\
\hline $\begin{array}{l}\text { Ankle Plantarflexion } \\
\text { TTP }\end{array}$ & $1.00(0.996-1.003$ & 0.90 \\
\hline Knee Flexion TTP & $1.00(0.998-1.002)$ & 0.86 \\
\hline Knee Extension TTP & $0.999(0.997-1.001)$ & 0.38 \\
\hline Hip Flexion TTP & $1.002(0.999-1.004)$ & 0.22 \\
\hline Hip Extension TTP & $1.005(1.000-1.009)$ & 0.04 \\
\hline \multicolumn{3}{|l|}{$\begin{array}{l}\text { Motor Control Test } \\
\text { Latencies }\end{array}$} \\
\hline BWS & $1.02(0.98-1.07)$ & 0.23 \\
\hline BWM & $1.01(0.96-1.05)$ & 0.68 \\
\hline BWL & $1.01(0.98-1.04)$ & 0.42 \\
\hline FWS & $1.01(0.98-1.03)$ & 0.42 \\
\hline FWM & $0.99(0.98-1.01)$ & 0.54 \\
\hline FWL & $1.01(0.98-1.03)$ & 0.44 \\
\hline
\end{tabular}

$\mathrm{DF}=$ Dorsiflexion; $\mathrm{PF}=$ Plantarflexion; TTP $=$ Time to

Peak BWS $=$ Backwards Small; BWM $=$ Backwards

Medium; BWL = Backwards Large; FWS = Forwards Small;

FWM $=$ Forwards Medium; FWL $=$ Forwards Large. ${ }^{\dagger}$ Reference group was recovering after the induced slip

Table 2. Multivariable logistic regression examining the association between recovery parameters and the odds of falling after an induced slip

\begin{tabular}{|c|c|c|}
\hline & \multicolumn{2}{|c|}{$\begin{array}{c}\text { Odds ratio }(95 \% \mathrm{CI}) \text { for } \\
\text { falling after the induced slip } \\
\text { perturbation }\end{array}$} \\
\hline & Odds ratio $(95 \% \mathrm{CI})$ & $\mathbf{p}$ \\
\hline \multicolumn{3}{|l|}{ Recovery Response } \\
\hline Average Ankle Moment & $0.001(0.001-0.005)$ & 0.002 \\
\hline $\begin{array}{l}\text { Ankle Dorsiflexion } \\
\text { TTP }\end{array}$ & $1.005(1.001-1.009)$ & 0.015 \\
\hline Hip Extension TTP & $1.006(1.000-1.011)$ & 0.041 \\
\hline
\end{tabular}

Table 3. Binary logistic regression unadjusted estimates examining the association between recovery parameters and the odds of experiencing a hazardous slip

Odds ratio $(95 \% \mathrm{CI})$ for having a hazardous slip

Odds ratio $(95 \% \mathrm{CI}) \quad$ p

\begin{tabular}{|c|c|c|}
\hline \multicolumn{3}{|l|}{ Recovery Response } \\
\hline $\begin{array}{l}\text { Average Ankle } \\
\text { Moment }\end{array}$ & $0.01(0.01-0.01)$ & $<0.001$ \\
\hline Average Knee Moment & $1.52(0.48-4.81)$ & 0.47 \\
\hline Average Hip Moment & $0.80(0.40-1.56)$ & 0.51 \\
\hline Ankle DF Peak & $0.90(0.46-1.74)$ & 0.75 \\
\hline Ankle PF Peak & $0.01(0.01-0.13)$ & 0.001 \\
\hline Knee Flexion Peak & $2.73(0.74-10.04)$ & 0.13 \\
\hline Knee Extension Peak & $1.03(0.67-1.58)$ & 0.88 \\
\hline Hip Flexion Peak & $1.19(0.89-1.60)$ & 0.22 \\
\hline Hip Extension Peak & $0.77(0.53-1.13)$ & 0.19 \\
\hline $\begin{array}{l}\text { Ankle Dorsiflexion } \\
\text { TTP }\end{array}$ & $1.002(0.999-1.006)$ & 0.15 \\
\hline $\begin{array}{l}\text { Ankle Plantarflexion } \\
\text { TTP }\end{array}$ & $1.00(0.996-1.004)$ & 0.94 \\
\hline Knee Flexion TTP & $0.999(0.996-1.001)$ & 0.26 \\
\hline Knee Extension TTP & $0.998(0.996-1.000)$ & 0.10 \\
\hline Hip Flexion TTP & $1.002(0.999-1.004)$ & 0.15 \\
\hline Hip Extension TTP & $1.005(1.000-1.009)$ & 0.05 \\
\hline \multicolumn{3}{|l|}{$\begin{array}{l}\text { Motor Control Test } \\
\text { Latencies }\end{array}$} \\
\hline BWS & $1.02(0.98-1.07)$ & 0.26 \\
\hline BWM & $1.02(0.97-1.06)$ & 0.35 \\
\hline BWL & $1.02(0.98-1.05)$ & 0.30 \\
\hline FWS & $1.01(0.99-1.04)$ & 0.19 \\
\hline FWM & $1.01(0.98-1.01)$ & 0.92 \\
\hline FWL & $1.02(0.99-1.04)$ & 0.20 \\
\hline
\end{tabular}

$\mathrm{DF}=$ Dorsiflexion; $\mathrm{PF}=$ Plantarflexion; TTP $=$ Time to

Peak; BWS = Backwards Small; BWM = Backwards

Medium; BWL = Backwards Large; FWS = Forwards Small;

FWM $=$ Forwards Medium; FWL $=$ Forwards Large.

$\dagger$ Reference group were those who experienced a non-hazardous slip

the hazardous slips compared to non-hazardous, as well as the knee extension peak moment $(p=0.09)$, which was increased in the hazardous slips compared to non-hazardous.

The unadjusted logistic regression coefficients for slip severity are shown in Table 3 . These estimates, along with the findings from the t-tests above were used to create the multivariable logistic regression. In Table 4, the multivariable logistic regression association between the recovery parameters and odds of hazardous slipping are shown. This final multivariable logistic regression classified recoveries, and falls correctly $91.7 \%$, and $77.8 \%$, respectively $\left(X^{2}=37.876\right.$, $p<0.001$, Nagelkerke $\left.\mathrm{R}^{2}=0.607\right)$. The results from this model suggest that as the average ankle moment increases in the slip period, the odds of experiencing a hazardous slip 
Table 4. Multivariable logistic regression examining the association between recovery parameters and the odds of experiencing a hazardous slip

\begin{tabular}{lcc}
\hline & $\begin{array}{c}\text { Odds ratio (95\% CI) for having } \\
\text { a hazardous slip }\end{array}$ \\
\cline { 2 - 3 } & \multicolumn{1}{c}{ Odds ratio (95\% CI) } & p \\
\hline $\begin{array}{l}\text { Recovery Response } \\
\text { Average Ankle }\end{array}$ & $0.01(0.00-0.01)$ & $<0.001$ \\
Moment & $1.007(1.000-1.013)$ & 0.03 \\
Hip Extension TTP & $1.001(0.997-1.004)$ & 0.64 \\
Knee Extension TTP & \multicolumn{2}{|}{} \\
\hline $\begin{array}{l}\text { TTP = Time to Peak. } \\
\text { †Reference group was recovering after the induced slip }\end{array}$ &
\end{tabular}

decrease. Further, as the time to peak hip extension and knee extension moments increase, the odds of experiencing a hazardous slip increase (Table 4).

\section{DISCUSSION}

The current study examined slip recovery responses of the lower extremity, as well as response times to static postural perturbations in hopes of isolating specific mechanisms that lend to increased odds of recovering after a slip. Our results suggest that the primary recovery variables of interest associated with odds of falling were the time to peak hip extension moment, time to peak ankle dorsiflexion moment and the average moment of the ankle over stance phase. Previous research by Cham and colleagues (2001) provided evidence of a primary recovery response to slip events that consist of knee flexion, and hip extension (Cham \& Redfern, 2001). They also suggested that these recovery responses were observed approximately $190 \mathrm{~ms}$ into stance phase. Our data supported these findings, with a slower hip response being associated with increased odds of falling. Indeed, the average time to peak hip extension moment was $117 \mathrm{~ms}$, and $190 \mathrm{~ms}$ for recoveries and falls, respectively.

Our findings at the ankle may even be representative of this hip response as well. While previous research has suggested that the ankle joint is relatively passive during the recovery response (Cham \& Redfern, 2001; Chambers \& Cham, 2007; O'Connell, Chambers, Mahboobin, \& Cham, 2016), it does appear important to maintain the center of pressure near the slipping heel in order to prolong weight transfer to this lead limb. We pose that our findings represent an inadequate primary response in the fallers, resulting in a prolonged time to peak ankle dorsiflexion moment. While the ankle itself per se, may not be actively involved in this recovery, it may be beneficial for future studies to combine these ankle kinetic profiles with traditional slip measures to aid in the classification of slip severity and fall status if newer harness load cell methods are unavailable to quantify falls.

Previous work by Lockhart et al. (2010) has also suggested that lower extremity strength and response times during the MCT were associated with increased slip distance in older adults (Lockhart, Smith, \& Woldstad, 2005). Our response time results also suggest an association with increased odds of experiencing a hazardous slip. However, they appeared less associated with the slip outcome. This may suggest that these automatic postural responses are associated more with less hazardous slips, that is often not perceived by the individual and do not require large gait modifications (Chander et al., 2015b; Redfern et al., 2001). Similar to our fall status model, we saw positive associations of hip extension time to peak with hazardous slips, as well as knee extension time to peak associations. The hip extension previously discussed as a primary recovery response appears to be a key component in the slip recovery response due to its association with both fall status, and slip severity classification. The knee extension moment has been previously observed as a secondary recovery response and thought to be involved in supporting the knee from buckling during the slip, and continuing forward locomotion (Cham \& Redfern, 2001; Chambers \& Cham, 2007). With a primary purpose of this knee extension suggested as continuing forward progress, it is likely this positive association in time to peak knee extension and hazardous slips are due to more non-hazardous slips resulting in recovery and continuing forward.

With slip training programs being used to help adults at risk of falls learn movements directly related to recovery responses (Parijat \& Lockhart, 2012), we hope the findings herein will aid slip perturbation training moving forward. Slip perturbation training has shown short term improvements in reactive slip adjustments including a reduction in muscle onset and time to peak activations of knee flexors and ankle plantar flexors, reduced ankle and knee coactivation, reduced slip displacement, and reduced time to peak knee flexion, trunk flexion, and hip flexion velocities. Moving forward, slip training studies that show beneficial effects of training in reducing slip severity, may also utilize our findings to target training programs to reactive responses most associated with fall outcomes such as the primary hip recovery response.

Limitations of this study, like other lab based fall studies, include participant's potential awareness of the possibility of slipping and falling and could adopt a more cautious gait pattern. However, we did not observe any significant changes in gait kinetics across normal gait trials, to suggest any gait alterations. Further, the methods employed have been used previously by our lab (Chander et al., 2015a, 2015b; Chander, Wade, et al., 2016), and others (Cham \& Redfern, 2001; Chambers \& Cham, 2007; Merrill, Chambers, \& Cham, 2017; O'Connell et al., 2016). While there are various strategies for slip recovery, our findings suggest that the primary recovery mechanism at the slipping hip may play a vital role in preventing the fall. Future research may utilize these findings to aid in slip perturbation training and decrease slip and fall risks.

\section{CONCLUSIONS}

During the slip recovery response, our results suggest that the primary recovery mechanism at the slipping hip may play a vital role in preventing the fall. These results may further benefit promising results seen from slip perturbation 
training studies and provide avenues of targeted slip training programs to specific reactive responses. Improvements in existing perturbation training could benefit multiple populations at increased risk of a fall, such as older adults, as well as younger or middle-aged adults in occupational settings. Finally, findings from the current study may aid in other labbased fall research which isn't able to utilize force/load metrics in the harness system.

\section{REFERENCES}

Adams PE, Martinez ME, Vickerie JL, Kirzinger WK. Summary health statistics for the U.S. population: National Health Interview Survey, 2010. Vital Health Stat 10. 2011 Dec;(251):1-117. PubMed PMID: 22624451.

Burns, E. R., Stevens, J. A., \& Lee, R. (2016). The direct costs of fatal and non-fatal falls among older adultsUnited States. Journal of safety research, 58, 99-103.

Bursac, Z., Gauss, C. H., Williams, D. K., \& Hosmer, D. W. (2008). Purposeful selection of variables in logistic regression. Source code for biology and medicine, 3(1), 17.

Cham, R., \& Redfern, M. S. (2001). Lower extremity corrective reactions to slip events. Journal of biomechanics, 34(11), 1439-1445.

Chambers, A. J., \& Cham, R. (2007). Slip-related muscle activation patterns in the stance leg during walking. Gait \& posture, 25(4), 565-572.

Chander, H., Garner, J. C., \& Wade, C. (2015a). Ground Reaction Forces in Alternative Footwear during Slip Events. International Journal of Kinesiology \& Sports Science, 3(2), 1.

Chander, H., Garner, J. C., \& Wade, C. (2015b). Heel contact dynamics in alternative footwear during slip events. International Journal of Industrial Ergonomics, 48, 158-166.

Chander, H., Garner, J. C., \& Wade, C. (2016). Slip outcomes in firefighters: A comparison of rubber and leather boots. Occupational Ergonomics, 13(2), 67-77.

Chander, H., Wade, C., Garner, J. C., \& Knight, A. C. (2016). Slip initiation in alternative and slip-resistant footwear. International journal of occupational safety and ergonomics, 1-12.
Chang, W.-R., Leclercq, S., Lockhart, T. E., \& Haslam, R. (2016). State of science: occupational slips, trips and falls on the same level. Ergonomics, 59(7), 861-883.

Kosma, M., Hondzinski, J. M., \& Buchanan, D. R. (2017). Exercise, health, and falls risks among older African American women. International Journal of Kinesiology and Sports Science, 5(3), 16-27.

Lee, A., Bhatt, T., Liu, X., Wang, Y., \& Pai, Y.-C. (2018). Can higher training practice dosage with treadmill slip-perturbation necessarily reduce risk of falls following overground slip? Gait \& posture, 61, 387-392.

Lockhart, T. E., Smith, J. L., \& Woldstad, J. C. (2005). Effects of aging on the biomechanics of slips and falls. $\mathrm{Hu}$ man factors, 47(4), 708-729.

Merrill, Z., Chambers, A. J., \& Cham, R. (2017). Arm reactions in response to an unexpected slip-impact of aging. Journal of biomechanics.

O’Connell, C., Chambers, A., Mahboobin, A., \& Cham, R. (2016). Effects of slip severity on muscle activation of the trailing leg during an unexpected slip. Journal of electromyography and kinesiology, 28, 61-66.

Parijat, P., \& Lockhart, T. E. (2012). Effects of moveable platform training in preventing slip-induced falls in older adults. Annals of biomedical engineering, 40(5), 1111-1121.

Redfern, M. S., Cham, R., Gielo-Perczak, K., Grönqvist, R., Hirvonen, M., Lanshammar, H., Powers, C. (2001). Biomechanics of slips. Ergonomics, 44(13), 1138-1166.

Sadeghi, H., Hakim, M. N., Hamid, T. A., Amri, S. B., Razeghi, M., Farazdaghi, M., \& Shakoor, E. (2017). The effect of exergaming on knee proprioception in older men: A randomized controlled trial. Archives of gerontology and geriatrics, 69, 144-150.

Sawers, A., Pai, Y.-C. C., Bhatt, T., \& Ting, L. H. (2017). Neuromuscular responses differ between slip-induced falls and recoveries in older adults. Journal of neurophysiology, 117(2), 509-522.

Strandberg, L., \& Lanshammar, H. (1981). The dynamics of slipping accidents. Journal of Occupational Accidents, 3(3), 153-162.

Yang, F., \& Pai, Y.-C. (2011). Automatic recognition of falls in gait-slip training: Harness load cell based criteria. Journal of biomechanics, 44(12), 2243-2249. 